\title{
Enumeration of perfect matchings of a type of quadratic lattice on the torus*
}

\author{
Fuliang Lu Lianzhu Zhang ${ }^{\dagger}$ Fenggen Lin \\ School of Mathematical Sciences, Xiamen University \\ Xiamen 361005, P. R. China. \\ Zhanglz@xmu.edu.cn \\ Submitted: Dec 6, 2009; Accepted: Feb 17, 2010; Published: Mar 8, 2010 \\ Mathematics Subject Classifications: 05A15, 05C30
}

\begin{abstract}
A quadrilateral cylinder of length $m$ and breadth $n$ is the Cartesian product of a $m$-cycle(with $m$ vertices) and a $n$-path(with $n$ vertices). Write the vertices of the two cycles on the boundary of the quadrilateral cylinder as $x_{1}, x_{2}, \cdots, x_{m}$ and $y_{1}, y_{2}, \cdots, y_{m}$, respectively, where $x_{i}$ corresponds to $y_{i}(i=1,2, \ldots, m)$. We denote by $Q_{m, n, r}$, the graph obtained from quadrilateral cylinder of length $m$ and breadth $n$ by adding edges $x_{i} y_{i+r}$ ( $r$ is a integer, $0 \leqslant r<m$ and $i+r$ is modulo $m$ ). Kasteleyn had derived explicit expressions of the number of perfect matchings for $Q_{m, n, 0}$ [P. W. Kasteleyn, The statistics of dimers on a lattice I: The number of dimer arrangements on a quadratic lattice, Physica 27(1961), 1209-1225]. In this paper, we generalize the result of Kasteleyn, and obtain expressions of the number of perfect matchings for $Q_{m, n, r}$ by enumerating Pfaffians.
\end{abstract}

Keywords: Pfaffian; Perfect matching; Quadratic lattice; Torus.

\section{Introduction}

The graphs considered in this paper have no loops or multiple edges. A perfect matching of a graph $G$ is a set of independent edges of $G$ covering all vertices of $G$. Problems involving enumeration of perfect matchings of a graph were first examined by chemists and physicists in the 1930s (for history see $[1,17]$ ), for two different (and unrelated) purposes: the study of aromatic hydrocarbons and the attempt to create a theory of the liquid state. Many mathematicians, physicists and chemists have given most of their attention to counting perfect matchings of graphs. See for example papers [5,6,12-15,21-23].

${ }^{*}$ This work is supposed by NFSC (NO.10831001).

${ }^{\dagger}$ Corresponding author 


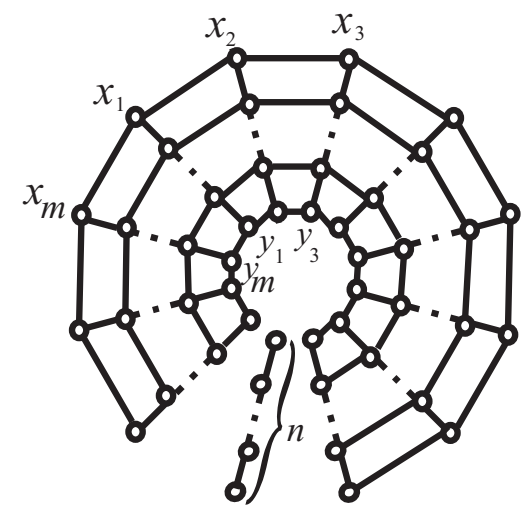

Figure 1: A quadrilateral cylinder circuit of length $m$ and breadth $n$.

\begin{tabular}{|c|c|c|c|c|}
\hline $\mathrm{r}$ & 0 & 1 & 2 & 3 \\
\hline$w_{Q_{6,6, r}}$ & 90176 & 63558 & 88040 & 64152 \\
\hline
\end{tabular}

Table 1: The number of perfect matchings of $Q_{6,6, r}$.

How many perfect matching does a given graph have? In general graphs, it is NP-hard. But for some special classes of graph, it can be solved exactly, especial to lattices(maybe infinite), such as the quadratic lattice, hexagonal lattice, triangular lattice, kagome lattice and etc[3,7,13,22]. For graph on torus, D. J. Klein[9] had considered finite-sized elemental benzenoid graphs corresponding to hexagonal. A quadrilateral cylinder of length $m$ and breadth $n$ is the Cartesian product of a $m$-cycle(with $m$ vertices) and a $n$-path(with $n$ vertices). Write the vertices of the two cycles on the boundary of the quadrilateral cylinder as $x_{1}, x_{2}, \cdots, x_{m}$ and $y_{1}, y_{2}, \cdots, y_{m}$, respectively, where $x_{i}$ corresponds to $y_{i}(i=$ $1,2, \ldots, m)$ (as indicated in Figure 1). We denote by $Q_{m, n, r}$, the graph obtained from quadrilateral cylinder of length $m$ and breadth $n$ by adding edges $x_{i} y_{i+r}(i=1,2, \ldots, m$, $r$ is a integer, $0 \leqslant r<m$ and $i+r$ is modulo $m$ ). Then the 4-regular graph $Q_{m, n, r}$ has natural embeddings on the torus[20].

If both $m$ and $n$ are odd, obviously, $Q_{m, n, r}$ does not have perfect matching. So, we suppose that at least one of $m$ and $n$ is even. Denote by $w_{Q_{m, n, r}}$, the number of perfect matchings of $Q_{m, n, r}$. Generally speaking, $w_{Q_{m, n, r}}$ is influenced by the value of $r$ (see Table 1).

Kasteleyn had discussed $Q_{m, n, 0}$, the quadratic lattice on torus(with periodic boundary conditions) in [7], and deduced an explicit expressions:

$$
\begin{aligned}
w_{Q_{m, n, 0}}= & \frac{1}{2} \prod_{k=1}^{n / 2} \prod_{l=1}^{m}\left(4 \sin ^{2} \frac{2 k \pi}{n}+4 \sin ^{2} \frac{2 l-1}{m} \pi\right)^{\frac{1}{2}}+\frac{1}{2} \prod_{k=1}^{n / 2} \prod_{l=1}^{m}\left(4 \sin ^{2} \frac{2 k-1}{n} \pi+4 \sin ^{2} \frac{2 l}{m} \pi\right)^{\frac{1}{2}} \\
& +\frac{1}{2} \prod_{k=1}^{n / 2} \prod_{l=1}^{m}\left(4 \sin ^{2} \frac{2 k-1}{n} \pi+4 \sin ^{2} \frac{2 l-1}{m} \pi\right)^{\frac{1}{2}} .
\end{aligned}
$$

He also stated that perfect matchings in a graph embedding on a surface of genus $g$ could 
be enumerated as a linear combination of $4^{g}$ Pfaffians of modified adjacency matrices of the graph, which was proved by Galluccio and loebl[4], Tesler[19], independently.

In this article, we generalize the result of Kasteleyn, and obtain expressions of $w_{Q_{m, n, r}}$, by enumerating Pfaffians. In section 2, we introduce the method of Tesler, and orient $Q_{m, n, r}$ by the crossing orientation rule. In section 3, we enumerate the number of perfect matchings of $Q_{m, n, r}$, by applying Tesler's method.

\section{Tesler's method and A crossing orientation of $Q_{m, n, r}$}

The Pfaffian method enumerating the number of different perfect matchings was independently discovered by Fisher[3], Kasteleyn[7], and Temperley [14]. See [11] for further details.

Given an undirected graph $G=(V(G), E(G))$ with vertex set $V(G)=\{1,2, \ldots, 2 p\}$, we allow each edge $\{i, j\}$ to have a weight $w_{\{i, j\}}$. To unweighted graphs, set weight to 1 for all edges. Let $G^{e}$ be an arbitrary orientation of $G$. Denote the arc of $G^{e}$ by $(i, j)$ if the direction of it is from $i$ to $j$. The skew adjacency matrix of $G^{e}$, denoted by $A\left(G^{e}\right)$, is defined as follows:

$$
A\left(G^{e}\right)=\left(a_{i j}\right)_{2 p \times 2 p}
$$

where

$$
a_{i j}=\left\{\begin{array}{cc}
w_{\{i, j\}} & \text { if }(i, j) \text { is an arc of } G^{e}, \\
-w_{\{i, j\}} & \text { if }(j, i) \text { is an arc of } G^{e}, \\
0 & \text { otherwise. }
\end{array}\right.
$$

Let $P M=\left\{\left\{i_{1}, i_{1}^{\prime}\right\}, \ldots,\left\{i_{p}, i_{p}^{\prime}\right\}\right\}$ range over the partitions of $1, \ldots, 2 p$ into $p$ sets of size 2 , and define the signed weight of $P M$ as

$$
w_{P M}=\operatorname{sign}\left(\begin{array}{ccccc}
1 & 2 & \cdots & 2 p-1 & 2 p \\
i_{1} & i_{1}^{\prime} & \cdots & i_{p} & i_{p}^{\prime}
\end{array}\right) \cdot a_{i_{1} i_{1}^{\prime}} \cdots a_{i_{p} i_{p}^{\prime}},
$$

(where the sign is of the permutation expressed in 2-line notation). The Pfaffian of $A$ is defined as

$$
\operatorname{Pf} A=\sum_{P M} w_{P M}
$$

Theorem 1 (The Cayley's Theorem, [11]). Let $A=\left(a_{i j}\right)_{2 p \times 2 p}$ be a skew symmetric matrix of order of $2 p$. Then the determinant of $A$, $\operatorname{det}(A)=(\operatorname{Pf} A)^{2}$.

When $P M$ is a partition that is not a perfect matching, $w_{P M}=0$, so the nonzero terms of $\operatorname{Pf} A$ correspond to the perfect matchings of $G$. We call $w_{P M}$ the signed weight of the perfect matching $P M$ and define the sign of $P M$ to be the sign of $w_{P M}$. If the signs of all the perfect matching of $G$ are the same, we say the orientation is Pfaffian orientation. A graph is Pfaffian if it has a Pfaffian orientation. Unfortunately, no polynomial algorithm is known for checking whether or not a given orientation of a graph $G$ is Pfaffian. 
Any compact boundaryless 2-dimensional surface $S$ can be represented in the plane by a plane model[19]. Draw a $2 l$ sided polygon $P$, and form $l$ pairs of sides $p_{j}, p_{j}^{\prime}, j=1, \ldots, l$. Paste together $p_{j}$ and $p_{j}^{\prime}$. Any $S$ can be represented by a suitable polygon and pastings. Introduce symbols $a_{1}, \ldots, a_{l}$. If $p_{j}$ and $p_{j}^{\prime}$ are pasted together by traversing $P$ clockwise along both, then place the label $a_{j}$ along both $p_{j}$ and $p_{j}^{\prime}$, and say that $S$ is $j$-nonoriented. If they are pasted by traversing $P$ clockwise along one and counterclockwise along the other, label the clockwise one $a_{j}$, the counterclockwise one $a_{j}^{-1}$, and say that $S$ is $j$-oriented. Form a word $\sigma$ from these $2 l$ symbols by starting at any side and read off the labels as $P$ is traversed clockwise. If the occurrences of $a_{j}$ or $a_{j}^{-1}$ are interleaved with the occurrences of $a_{k}$ or $a_{k}^{-1}$, such as in $\sigma=\ldots a_{j} \ldots a_{k}^{-1} \ldots a_{j} \ldots a_{k} \ldots$, we say that $\sigma$ is $j, k$-alternating; otherwise it is $j, k$-nonalternating. Now take an embedding of a graph $G$ on this surface, and draw it within this plane model of the surface. Edges wholly contained inside the polygon $P$ do not cross, and are called 0-edges. The edges that go through sides $p_{j}, p_{j}^{\prime}$ of $P$ are called $j$-edges. We say a face of a planar graph is clockwise odd when it has an odd number of edges pointing along its boundary when traversed clockwise.

Introduce new variables $x_{1}, \ldots, x_{l}$. Multiply the weights of all $j$-edges by $x_{j}(j \neq 0)$, and let $B\left(x_{1}, \ldots, x_{l}\right)$ be the $x$-adjacency matrix, with $b_{u v}=a_{u v}$ when $(u, v)$ is a 0-edge, while $b_{u v}=a_{u v} x_{j}$ when $(u, v)$ is a $j$-edge $(j \neq 0)$, where $a_{i j}$ is the entry of the $A\left(G^{e}\right)$, the skew adjacency matrix of $G^{e}$.

Let

$$
f\left(\omega_{1}, \ldots, \omega_{l}\right)=\sum_{0 \leqslant r_{1}, r_{2}, \ldots, r_{l} \leqslant 3} \alpha_{r_{1}, \ldots, r_{l}} \omega_{1}^{r_{1}} \ldots \omega_{l}^{r_{l}},
$$

all exponents of $\omega_{i}$ are to be reduced modulo 4 to one of $0,1,2,3$. We consider any perfect matching $P M$ in $G$. The $f$-weight of the perfect matching $P M$ is

$$
w_{P M}(f)=f\left(i^{N_{P M}(1)}, \ldots, i^{N_{P M}(l)}\right) w_{P M},
$$

where $N_{P M}(j)$ be the number of $j$-edges in $P M, i=\sqrt{-1}$. The $f$-weight of $G$ is

$$
\begin{aligned}
w_{G}(f) & =\sum_{r_{1}, r_{2}, \ldots, r_{l}} \alpha_{r_{1}, r_{2}, \ldots, r_{l}} \operatorname{PfB}\left(i^{r_{1}}, \ldots, i^{r_{l}}\right) \\
& =\sum_{r_{1}, r_{2}, \ldots, r_{l}} \alpha_{r_{1}, r_{2}, \ldots, r_{l}} \sum_{P M} w_{P M} \cdot i^{r_{1} N_{P M}(1)} \ldots i^{r_{l} N_{P M}(l)}=\sum_{P M} w_{P M}(f) .
\end{aligned}
$$

Theorem 2 [19]. The total unsigned weight of all perfect matchings in $G$ is

$$
\varepsilon_{0} w_{G}\left(\prod_{1 \leqslant j \leqslant k \leqslant l} L_{j k}\right)
$$

Where $\varepsilon_{0}= \pm 1, L_{j j}= \begin{cases}\frac{1-i}{2} \omega_{j}+\frac{1+i}{2} \omega_{j}^{-1} & \text { if } \sigma \text { is } j \text {-nonoriented; } \\ 1 & \text { otherwise. }\end{cases}$

$L_{j k}= \begin{cases}\frac{1}{2}\left(1+\omega_{j}^{2}+\omega_{k}^{2}-\omega_{j}^{2} \omega_{k}^{2}\right) & \text { if } \sigma \text { is } j, k \text {-alternating } \\ 1 & \text { otherwise. }\end{cases}$ 
Consider the graph $G$ embedded on torus. Letting $B\left(x_{1}, x_{2}\right)$ be its $x$-adjacency matrix, with the 0 -edges having weight 1 and the $j$-edges having weight $x_{j}(j=1,2)$,

$$
f=\prod_{1 \leqslant j \leqslant k \leqslant 2} L_{j k}=L_{11} L_{12} L_{22}=L_{12}=\frac{1}{2}\left(1+\omega_{1}^{2}+\omega_{2}^{2}-\omega_{1}^{2} \omega_{2}^{2}\right) .
$$

Thus, by Equation(2), the number of perfect matchings of $G$ is given by

$$
\pm w_{G}(f)=\frac{1}{2}[\operatorname{PfB}(1,1)+\operatorname{Pf} B(-1,1)+\operatorname{Pf} B(1,-1)-\operatorname{Pf} B(-1,-1)] .
$$

The graph $Q_{m, n, r}$ can be embedded on the torus, so we draw its planar subgraph con-

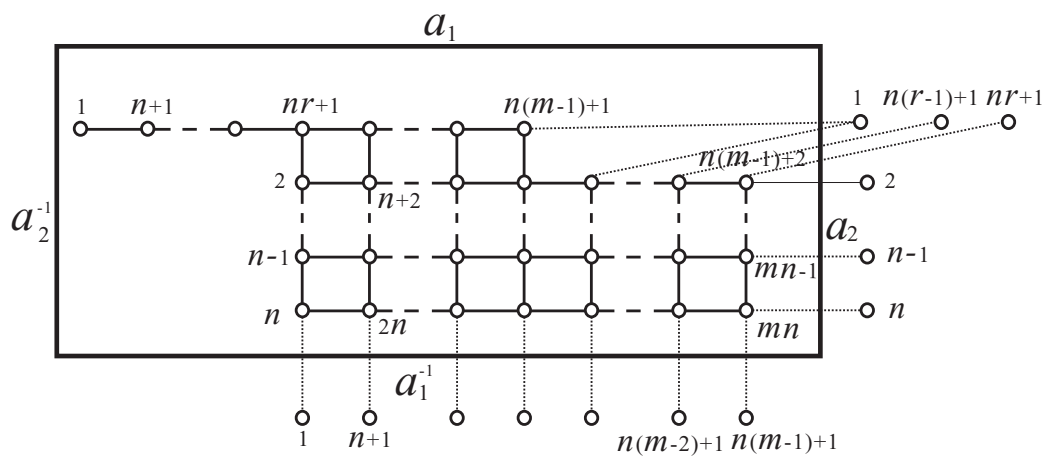

Figure 2: The subgraph of 0-edges of $Q_{m, n, r}$.

taining all vertices in a 4-polygon which is 1,2-alternating and label $m n$ vertices of $Q_{m, n, r}$ by $1,2, \cdots, m n$ shown in Figure 2 . Thus 0 -edges set $E_{0}, 1$-edges set $E_{1}$ and 2-edges set $E_{2}$ of $Q_{m, n, r}$, respectively, are

$E_{0}=\{\{k n+t,(k+1) n+t\} \mid k=0, \ldots, m-2, t=1, \ldots, n\} \cup\{\{k n+t, k n+t+1\} \mid k=$ $0, \ldots, m-1, t=2, \ldots, n-1\} \cup\{\{n(r+k)+1, k n+2\} \mid k=0, \ldots, m-1-r\}$, $E_{1}=\{\{k n,(k-1) n+1\} \mid k=1, \ldots, m\}$, $E_{2}=\{\{t, n(m-1)+t\} \mid t=1, \ldots, n\} \cup\{\{k n+1, n(m-r+k)+2\} \mid k=0, \ldots, r-1\}$.

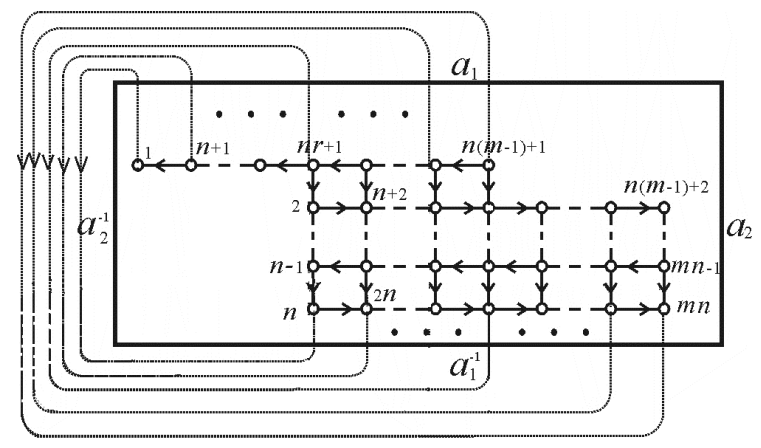

(a)

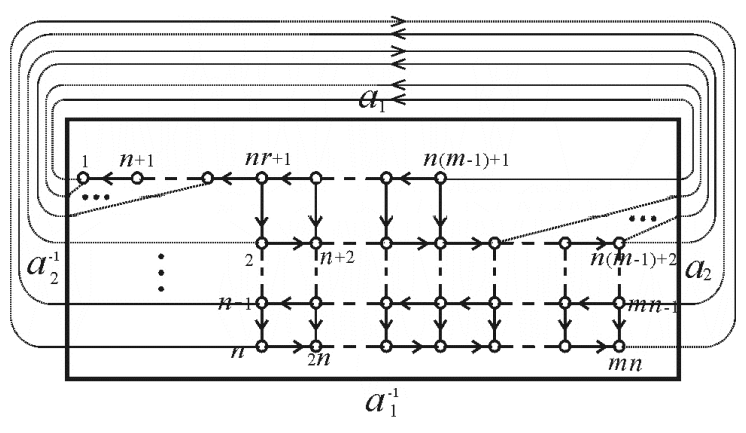

(b)

Figure 3: the orientation of $Q_{m, n, r}$ when $n$ is odd. 


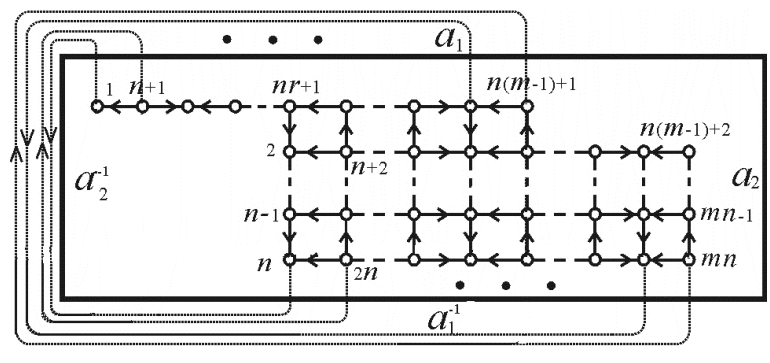

(a)

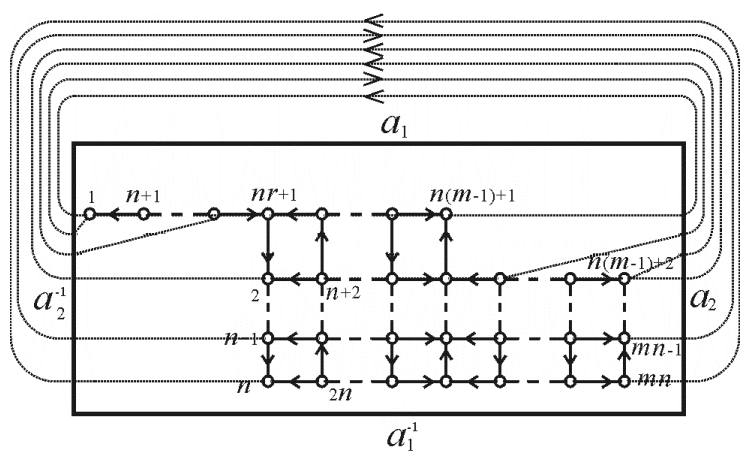

(b)

Figure 4: the orientation of $Q_{m, n, r}$ when $n$ is odd.

Crossing orientation rule [19]: Orient the subgraph of 0-edges so that all its faces are clockwise odd. Orient each $j$-edge $e(j>0)$ as follows. Ignoring all other non 0-edges, there is a face formed by $e$ and certain 0-edges along the boundary of the subgraph of 0 -edges. Orient e so that this face is clockwise odd.

When $n$ is even, for an edge $e=\{k, l\}$ of $Q_{m, n, r}$, without loss of generality, suppose $k<l$. If $e \in E_{0}$, orient it from $k$ to $l$ when both $k$ and $l$ are even or $k+1=l$, otherwise from $l$ to $k$, referring to Figure $3(a)$. If $e \in E_{1}$, let the direction of it be from $k$ to $l$. If $e \in E_{2}$, orient it from $k$ to $l$ when both $k$ and $l$ are even, otherwise from $l$ to $k$, for even $m$. Reversing the direction of $e$ when $m$ is odd(as in Figure 3(b)).

If $n$ is odd, $r$ is even, we orient the graph as Figure 4 shown. Figure $4(a)$ shows the direction of 1-edges when $r \equiv 2(\bmod 4)$, reversing the direction of all the 1-edges when $r \equiv 0(\bmod 4)$. When $m \equiv 2(\bmod 4)$, the direction of 2 -edges are shown in Figure $4(b)$, reversing when $m \equiv 0(\bmod 4)$.

Lemma 3 Suppose $Q_{m, n, r}^{e}$ is the orientation of $Q_{m, n, r}$ as above, then it is a crossing orientation.

Proof: It is easy to check that all the faces of the subgraph of 0-edges are clockwise odd. For 2-edges $\{k n+1, n(m-r+k)+2\} \mid k=0, \ldots, r-1\}$ when $m$ is even, the vertices of the cycle formed by one of them and certain 0-edges along the boundary of the subgraph of 0-edges are $[n(m-r+k)+2],[n(m-r-1)+2],[n(m-1)+1], \cdots,(n+1), 1,(n+$ $1), \cdots,(k n+1)$. The number of edges pointing along it from vertex $n(m-r+k)+2$ to $k n+1$ traversed clockwise always is odd, so does this cycle. Similar discussion solves the other case, and the lemma follows.

Theorem 4 [19]. (a)A graph may be oriented so that every perfect matching PM has sign $\epsilon_{P M}=\epsilon_{0}(-1)^{\kappa(P M)}$, where $\epsilon_{0}= \pm 1$ is constant; $\epsilon_{0}$ may be interpreted as the sign of a perfect matching with no crossing edges when such exists; $\kappa(P M)$ be the number of times edges in it cross.

(b) An orientation of a graph satisfies (a) if, and only if, it is a crossing orientation. 


\section{Enumeration of perfect matchings of $Q_{m, n, r}$}

\subsection{The sign of $p f B\left(x_{1}, x_{2}\right)$}

In order to decide the sign of pfaffians of $B\left(x_{1}, x_{2}\right)\left(x_{1}, x_{2}= \pm 1\right)$, we distinguish the perfect matchings of $Q_{m, n, r}$ into four classes. The perfect matchings belonging to class 1 are those that have odd number of 1-edges and odd number of 2-edges; The perfect matchings in class 2 have odd number of 1-edges and even number of 2-edges; The perfect matchings in class 3 have even number of 1-edges and odd number of 2-edges and the ones have even number of 1-edges and even number of 2-edges in class 4 . So except the perfect matching $P M$ in class 1 , the number of times edges in it cross $\kappa(P M)$ are always even.

Consider the case when $x_{1}=1$ and $x_{2}=1$ firstly. If $n$ is even, then obviously, the edges set $P M_{1}=\{\{1, n\},\{2,3\},\{4,5\}, \cdots,\{n-2, n-1\},\{n+1,2 n\},\{n+2, n+3\},\{n+4, n+$ $5\}, \cdots,\{2 n-2,2 n-1\}, \cdots,\{(m-1) n+1, m n\},\{(m-1) n+2,(m-1) n+3\},\{(m-1) n+$ $4,(m-1) n+5\}, \cdots,\{m n-2, m n-1\}\}$ is a perfect matching in class 2 or class 4 according to the parity of $m$. Note that $n$ is even and $x_{1}=1, x_{2}=1$, so $a_{1 n} a_{23} \cdots a_{(m n-2)(m n-1)}=1$ and

$$
\operatorname{sign}\left(\begin{array}{ccccccc}
1 & 2 & 3 & 4 & \cdots & m n-1 & m n \\
1 & n & 2 & 3 & \cdots & m n-2 & m n-1
\end{array}\right)=(-1)^{m(n-2)}=1 .
$$

Then the sign of $P M_{1}$ is positive.

If $n$ is odd. Let $P M_{2}=\{\{1, n+1\},\{2 n+1,3 n+1\}, \cdots,\{(m-2) n+1,(m-$ 1) $n+1\},\{2, n+2\},\{2 n+2,3 n+2\}, \cdots,\{(m-2) n+2,(m-1) n+2\}, \cdots,\{n, 2 n\}$, $\{3 n, 4 n\}, \cdots,\{(m-1) n, m n\}\}$, then $P M_{2}$ is a perfect matching of $Q_{m, n, r}$ which belongs to class 4 . Moreover,

$$
\begin{aligned}
& a_{1(n+1)} a_{(2 n+1)(3 n+1)} \cdots a_{(m n-n) m n}=(-1)^{m n / 2}, \\
& \operatorname{sign}\left(\begin{array}{ccccccc}
1 & 2 & 3 & 4 & \cdots & m n-1 & m n \\
1 & n+1 & 2 n+1 & 3 n+1 & \cdots & m n-n & m n
\end{array}\right)=(-1)^{\sum_{j=1}^{m-1} \sum_{i=1}^{n-1} i j} .
\end{aligned}
$$

Note that $(-1)^{\sum_{j=1}^{m-1} \sum_{i=1}^{n-1} i j}$ equals to 1 when $m \equiv 0(\bmod 4)$, equals to -1 when $m \equiv 2$ $(\bmod 4)$, so the sign of $P M_{2}$ also is positive. Then, by Theorem 4, the sign of perfect matchings in class 2, class 3 and class 4 is positive, except perfect matchings in class 1 .

If $x_{1}=-1$, note that the number of 1-edges in class 2 is odd and the number of times edges in class 2 cross always is even, by Theorem 4, the sign of the perfect matching in this class is negative. Similar discussion to the other cases, the signs of perfect matchings can be decided, as shown in Table 2 .

Lemma 5. If PfB(-1,1) or PfB(-1,-1) equals to zero, then $\operatorname{Pf} B(-1,-1) \leqslant 0$, $\operatorname{Pf} B(1,1) \geqslant 0, \operatorname{Pf} B(-1,1) \geqslant 0, \operatorname{Pf} B(1,-1) \geqslant 0$.

Proof: Denote the number of perfect matchings belonging to class $i(i=1,2,3,4)$ by $w_{i}$. If $\operatorname{PfB}(-1,1)=0$ then by Table 2 , that means $w_{2}=w_{1}+w_{3}+w_{4}$, so $w_{1}+w_{2}+w_{3} \geqslant w_{4}$. Notice that a perfect matching in class $i(i=1,2,3)$ contributes -1 to $\operatorname{Pf} B(-1,-1)$, so $\operatorname{Pf} B(-1,-1) \leqslant 0$. Similar discussion completes the other cases of the Lemma. 


\begin{tabular}{|c|c|c|c|c|}
\hline \multirow{2}{*}{ class } & \multicolumn{4}{|c|}{ sign of corresponding perfect matchings } \\
\cline { 2 - 5 } & $x_{1}=1, x_{2}=1$ & $x_{1}=-1, x_{2}=1$ & $x_{1}=1, x_{2}=-1$ & $x_{1}=-1, x_{2}=-1$ \\
\hline 1 & - & + & + & - \\
\hline 2 & + & - & + & - \\
\hline 3 & + & + & - & - \\
\hline 4 & + & + & + & + \\
\hline
\end{tabular}

Table 2: The signs of the perfect matchings

\subsection{Enumerate the perfect matchings of $Q_{m, n, r}^{e}$}

Recalled that $Q_{m, n, r}^{e}$ is a crossing orientation of $Q_{m, n, r}$, the $x$-adjacency matrix of $Q_{m, n, r}^{e}$, denoted by $B\left(x_{1}, x_{2}\right)$. Then the elements of $B\left(x_{1}, x_{2}\right)$ can be read off from Figure 3 or Figure 4, has the following form:

$$
B\left(x_{1}, x_{2}\right)=\left(B_{i j}\left(x_{1}, x_{2}\right)\right)
$$

where $B_{i j}\left(x_{1}, x_{2}\right)$ is the $n \times n$ matrix. If $n$ is even, when $j \geqslant i$,

$$
B_{i j}= \begin{cases}A\left(x_{1}\right) & \text { if } i=j, i=1, \ldots, m \\ B(-1) & \text { if } j=i+1, i=1, \ldots, m-1 \\ C(-1)^{T} & \text { if } j=i+r, i=1, \ldots, m-r \\ (-1)^{m+1} C\left(x_{2}\right) & \text { if } j=i+m-r, i=1, \ldots, r \\ (-1)^{m+1} B\left(x_{2}\right) & \text { if } i=1, j=m \\ 0_{n} & \text { otherwise. }\end{cases}
$$

When $j<i, B_{i j}=-B_{j i}^{T}\left(B_{i j}^{T}\right.$ is the transpose of $\left.B_{i j}\right)$. If $n$ is odd and $r$ is even,

$$
B_{i j}= \begin{cases}\epsilon A\left(-(-1)^{r / 2} x_{1}\right) & \text { if } i=j, i=1, \ldots, m ; \\ \epsilon B^{\prime}(-1) & \text { if } j=i+1, i=1, \ldots, m-1 \\ \epsilon C(-1)^{T} & \text { if } j=i+r, i=1, \ldots, m-r \\ \epsilon(-1)^{m / 2} C\left(-x_{2}\right) & \text { if } j=i+m-r, i=1, \ldots, r \\ \epsilon(-1)^{m / 2} B^{\prime}\left(x_{2}\right) & \text { if } i=1, j=m ; \\ 0_{n} & \text { otherwise. }\end{cases}
$$

When $j<i, B_{i j}=-B_{j i}^{T}\left(B_{i j}^{T}\right.$ is the transpose of $\left.B_{i j}\right)$, if $i$ is even, $\epsilon=-1$, else $\epsilon=1$. Where

$$
A(x)=\left[\begin{array}{cccccc}
0 & 0 & 0 & 0 & \cdots & x \\
0 & 0 & 1 & 0 & \cdots & 0 \\
0 & -1 & 0 & 1 & \cdots & 0 \\
\vdots & \vdots & \ddots & \ddots & \ddots & \vdots \\
0 & 0 & \cdots & -1 & 0 & 1 \\
-x & 0 & 0 & \cdots & -1 & 0
\end{array}\right], C(x)=\left[\begin{array}{cccc}
0 & x & 0 & \cdots \\
0 & 0 & 0 & \cdots \\
0 & 0 & 0 & \cdots \\
\vdots & \vdots & \vdots & \ddots
\end{array}\right]
$$




$$
B(x)=\left[\begin{array}{lllll}
x & & & & \\
& -x & & & \\
& & \ddots & & \\
& & & x & \\
& & & & -x
\end{array}\right], B^{\prime}(x)=\left[\begin{array}{lllll}
x & & & & \\
& x & & \\
& & \ddots & & \\
& & x & \\
& & & x
\end{array}\right],
$$

$0_{n}$ is a $n \times n$ matrix and all its entries are zero.

In order to calculate the determinant of $B\left(x_{1}, x_{2}\right)$, we introduce the following lemma. Firstly, denote the block circulant matrix

$$
\left(\begin{array}{cccc}
V_{0} & V_{1} & \cdots & V_{m-1} \\
V_{m-1} & V_{0} & \cdots & V_{m-2} \\
\vdots & \vdots & \ddots & \vdots \\
V_{1} & V_{2} & \cdots & V_{0}
\end{array}\right)
$$

by $\operatorname{circ}\left(V_{0}, V_{1}, \cdots, V_{m-1}\right)$, and denote the skew block circulant matrix

$$
\left(\begin{array}{ccccc}
V_{0} & V_{1} & V_{2} & \cdots & V_{m-1} \\
-V_{m-1} & V_{0} & V_{1} & \cdots & V_{m-2} \\
-V_{m-2} & -V_{n-1} & V_{0} & \cdots & V_{m-3} \\
\vdots & \vdots & \ddots & \ddots & \vdots \\
-V_{1} & -V_{2} & \cdots & -V_{m-1} & V_{0}
\end{array}\right)
$$

by $\operatorname{scirc}\left(V_{0}, V_{1}, \cdots, V_{m-1}\right)$.

Lemma 6 ([2]). Let $V=\operatorname{circ}\left(V_{0}, V_{1}, \cdots, V_{m-1}\right)$ or $V=\operatorname{scirc}\left(V_{0}, V_{1}, \cdots, V_{m-1}\right)$ be a block circulant matrix or a skew block circulant matrix over the complex number field, where all $V_{t}$ are $n \times n$ matrices, $t=0,1, \ldots, m-1$. Then

$$
\operatorname{det} V=\prod_{t=0}^{m-1} \operatorname{det}\left(J_{t}\right)
$$

where $J_{t}=V_{0}+V_{1} \omega_{t}+V_{2} \omega_{2 t}+\cdots+V_{m-1} \omega_{(m-1) t}$,

$$
\omega_{t}=\left\{\begin{array}{ll}
\cos \frac{2 t \pi}{m}+i \sin \frac{2 t \pi}{m} & (\text { if } V \text { is a block circulant matrix }) \\
\cos \frac{(2 t+1) \pi}{m}+i \sin \frac{(2 t+1) \pi}{m} & (\text { if } V \text { is a skew block circulant matrix })
\end{array} .\right.
$$

We consider the case when $n$ is even firstly. In fact, if $m$ is odd, $x_{2}=1$ or $m$ is even, $x_{2}=-1$, then

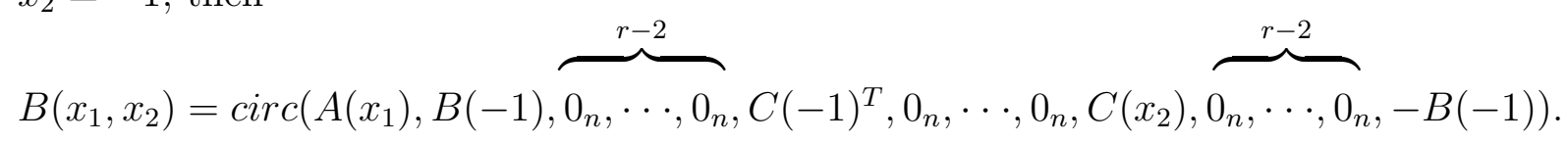


If $m$ is odd, $x_{2}=-1$ or $m$ is even, $x_{2}=1$, then

$B\left(x_{1}, x_{2}\right)=\operatorname{scirc}(A\left(x_{1}\right), B(-1), \overbrace{0_{n}, \cdots, 0_{n}}^{r-2}, C(-1)^{T}, 0_{n}, \cdots, 0_{n},-C\left(x_{2}\right), \overbrace{0_{n}, \cdots, 0_{n}}^{r-2} B(-1))$.

So by Lemma 6 , we always have that

$$
\operatorname{det}\left(B\left(x_{1}, x_{2}\right)\right)=\prod_{t=0}^{m-1} \operatorname{det}\left(F_{t}\right)
$$

where

$$
\begin{aligned}
& F_{t}=A\left(x_{1}\right)+\omega_{t} B(-1)-\omega_{t}^{-1} B(-1)+\omega_{r}^{-1} C(-1)^{T}+\omega_{r} C\left(x_{2}\right) \\
& =\left|\begin{array}{cccccc}
\beta & \omega_{r}^{-1} & & & & x_{1} \\
-\omega_{r} & -\beta & 1 & & & \\
& -1 & \beta & 1 & & \\
& & \ddots & \ddots & \ddots & \\
& & & -1 & \beta & 1 \\
-x_{1} & & & & -1 & -\beta
\end{array}\right| \quad\left(\beta=-\omega_{t}+\omega_{t}^{-1}, t=0,1, \ldots, m-1\right), \\
& \omega_{t}=\left\{\begin{array}{ll}
\cos \frac{2 t \pi}{m}+i \sin \frac{2 t \pi}{m} & \left(m \text { is odd, } x_{2}=1 \text { or } m \text { is even, } x_{2}=-1\right) \\
\cos \frac{(2 t+1) \pi}{m}+i \sin \frac{(2 t+1) \pi}{m} & \left(m \text { is odd, } x_{2}=-1 \text { or } m \text { is even, } x_{2}=1\right)
\end{array} .\right.
\end{aligned}
$$

Furthermore, $\operatorname{det}\left(F_{t}\right)$ can be simplify as: if $n \equiv 0(\bmod 4)$,

$$
\operatorname{det}\left(F_{t}\right)=\left(-\omega_{t}+\omega_{t}^{-1}\right) T_{n-1}-2 T_{n-2}+x_{1}\left(\omega_{t}^{r}+\omega_{t}^{-r}\right),
$$

if $n \equiv 2(\bmod 4)$,

$$
\operatorname{det}\left(F_{t}\right)=\left(\omega_{t}-\omega_{t}^{-1}\right) T_{n-1}+2 T_{n-2}+x_{1}\left(\omega_{t}^{r}+\omega_{t}^{-r}\right),
$$

Where

$$
\begin{aligned}
& T_{n}=\left|\begin{array}{ccccc}
\beta & 1 & & & \\
1 & \beta & 1 & & \\
& \ddots & \ddots & \ddots & \\
& & 1 & \beta & 1 \\
& & & 1 & \beta
\end{array}\right|=\prod_{k=1}^{n}\left(\left(-\omega_{t}+\omega_{t}^{-1}\right)+2 \cos \frac{k \pi}{n+1}\right) \\
& =\frac{\left(\left(\omega_{t}^{-1}-\omega_{t}\right)+\sqrt{\left(\omega_{t}^{-1}-\omega_{t}\right)^{2}-4}\right)^{n+1}-\left(\left(\omega_{t}^{-1}-\omega_{t}\right)-\sqrt{\left(\omega_{t}^{-1}-\omega_{t}\right)^{2}-4}\right)^{n+1}}{2^{n+1} \sqrt{\left(-\omega_{t}+\omega_{t}^{-1}\right)^{2}-4}} .
\end{aligned}
$$

When $m$ is odd, $x_{2}=1$ or $m$ is even, $x_{2}=-1$,

$$
-\omega_{t}+\omega_{t}^{-1}=-2 i \sin \frac{2 t \pi}{m}, \omega_{t}^{r}+\omega_{t}^{-r}=2 \cos \frac{2 r t \pi}{m} .
$$

Noticing that

$$
\prod_{k=1}^{n}\left(2 \cos \frac{k \pi}{n+1}\right)=(i)^{n}, \text { when } n \text { is even. }
$$


Therefore, $\operatorname{det}\left(F_{0}\right)=2+2 x_{1}$. That is, when $x_{1}=-1, \operatorname{det}\left(F_{0}\right)=0$. By Theorem 1 and Equation (3), when $m$ is odd, $(P f B(-1,1))^{2}=\operatorname{det}\left(A_{1}(-1,1)=0\right.$, when $m$ is even, $(\operatorname{Pf} B(-1,-1))^{2}=\operatorname{det}\left(A_{1}(-1,-1)=0\right.$. By Lemma $5, \operatorname{Pf} B(-1,-1)=$ $-\operatorname{det}\left(A_{1}(-1,-1)\right)^{1 / 2}, \operatorname{Pf} B(1,1)=\operatorname{det}\left(A_{1}(1,1)\right)^{1 / 2}, \operatorname{PfB}(1,-1)=\operatorname{det}\left(A_{1}(1,-1)\right)^{1 / 2}$, $\operatorname{Pf} B(-1,1)=\operatorname{det}\left(A_{1}(-1,1)\right)^{1 / 2}$. So, if $n \equiv 0(\bmod 4)$, the number of perfect matchings of $Q_{m, n, r}$ :

$$
\begin{aligned}
w_{Q}= & \frac{1}{2}\left[\prod_{t=0}^{m-1}\left(-2 i T_{n-1}^{0} \sin \frac{2 t \pi}{m}-2 T_{n-2}^{0}+2 \cos \frac{2 t r \pi}{m}\right)^{1 / 2}\right. \\
& +\prod_{t=0}^{m-1}\left(-2 i T_{n-1}^{1} \sin \frac{(2 t+1) \pi}{m}-2 T_{n-2}^{1}+2 \cos \frac{(2 t+1) r \pi}{m}\right)^{1 / 2} \\
& \left.+\prod_{t=0}^{m-1}\left(-2 i T_{n-1}^{1} \sin \frac{(2 t+1) \pi}{m}-2 T_{n-2}^{1}-2 \cos \frac{(2 t+1) r \pi}{m}\right)^{1 / 2}\right] ;
\end{aligned}
$$

If $n \equiv 2(\bmod 4)$,

$$
\begin{aligned}
w_{Q}= & \frac{1}{2}\left[\prod_{t=0}^{m-1}\left(2 i T_{n-1}^{0} \sin \frac{2 t \pi}{m}+2 T_{n-2}^{0}+2 \cos \frac{2 t r \pi}{m}\right)^{1 / 2}\right. \\
& +\prod_{t=0}^{m-1}\left(2 i T_{n-1}^{1} \sin \frac{(2 t+1) \pi}{m}+2 T_{n-2}^{1}+2 \cos \frac{(2 t+1) r \pi}{m}\right)^{1 / 2} \\
& \left.+\prod_{t=0}^{m-1}\left(2 i T_{n-1}^{1} \sin \frac{(2 t+1) \pi}{m}+2 T_{n-2}^{1}-2 \cos \frac{(2 t+1) r \pi}{m}\right)^{1 / 2}\right],
\end{aligned}
$$

where $T_{n}^{0}=\prod_{k=1}^{n}\left(-2 i \sin \frac{2 t \pi}{m}+2 \cos \frac{k \pi}{n+1}\right), T_{n}^{1}=\prod_{k=1}^{n}\left(-2 i \sin \frac{(2 t+1) \pi}{m}+2 \cos \frac{k \pi}{n+1}\right)$. So we have

Theorem 7 If $n$ is even, then the number of perfect matchings of $Q_{m, n, r}$,

$$
w_{Q}=\frac{1}{2}\left\{\prod_{t=0}^{m-1}\left[H_{1}\left(\frac{2 t \pi}{m}\right)\right]^{\frac{1}{2}}+\prod_{t=0}^{m-1}\left[H_{1}\left(\frac{2 t+1}{m} \pi\right)\right]^{\frac{1}{2}}+\prod_{t=0}^{m-1}\left[H_{2}\left(\frac{2 t+1}{m} \pi\right)\right]^{\frac{1}{2}}\right\} .
$$

Where

$$
\begin{aligned}
& H_{1}(\theta)=\left(\sqrt{1+\sin ^{2} \theta}-\sin \theta\right)^{n}+\left(\sqrt{1+\sin ^{2} \theta}+\sin \theta\right)^{n}+2 \operatorname{cosr} \theta \\
& H_{2}(\theta)=\left(\sqrt{1+\sin ^{2} \theta}-\sin \theta\right)^{n}+\left(\sqrt{1+\sin ^{2} \theta}+\sin \theta\right)^{n}-2 \cos \theta
\end{aligned}
$$

If $n$ is odd and $m, r$ is even, multiplying $B_{i j}\left(x_{1}, x_{2}\right)$ by -1 when $i$ is even, a block circulant matrix or a skew block circulant matrix can be gotten. With the same discussion as above to the case, we have:

Theorem 8 If $n$ is odd and $m, r$ is even, then the number of perfect matchings of $Q_{m, n, r}$,

$$
w_{Q}=\left\{\begin{array}{lll}
\frac{1}{2} \sqrt{\prod_{t=0}^{m-1}\left[G_{1}\left(\frac{2 t+1}{m} \pi\right)\right.}+\frac{1}{2} \sqrt{\prod_{t=0}^{m-1}\left[G_{2}\left(\frac{2 t+1}{m} \pi\right)\right.} & (\text { if } m \equiv 0 & (\bmod 4)), \\
\frac{1}{2} \sqrt{-\prod_{t=0}^{m-1} G_{1}\left(\frac{2 t}{m} \pi\right)}+\frac{1}{2} \sqrt{-\prod_{t=0}^{m-1} G_{2}\left(\frac{2 t}{m} \pi\right)} & (\text { if } m \equiv 2 & (\bmod 4)) .
\end{array}\right.
$$


Where

$$
\begin{aligned}
& G_{1}(\theta)=\left(\sqrt{1+\cos ^{2} \theta}-\cos \theta\right)^{n}-\left(\sqrt{1+\cos ^{2} \theta}+\cos \theta\right)^{n}+2 i \operatorname{sinr} \theta, \\
& G_{2}(\theta)=\left(\sqrt{1+\cos ^{2} \theta}-\cos \theta\right)^{n}-\left(\sqrt{1+\cos ^{2} \theta}+\cos \theta\right)^{n}-2 i \operatorname{sinr} \theta .
\end{aligned}
$$

\section{Concluding remarks}

1. As a special case, when $r=0$, with the aid of the following identity, valid for even $n$ :

$$
\begin{gathered}
\left\{\left[u+\left(1+u^{2}\right)^{\frac{1}{2}}\right]^{n}+\left[-u+\left(1+u^{2}\right)^{\frac{1}{2}}\right]^{n}+2\right\}^{\frac{1}{2}}=\prod_{k=0}^{\frac{n}{2}-1} 2\left(u^{2}+\sin ^{2} \frac{2 k+1}{n} \pi\right)^{\frac{1}{2}}, \\
\left\{\left[u+\left(1+u^{2}\right)^{\frac{1}{2}}\right]^{n}+\left[-u+\left(1+u^{2}\right)^{\frac{1}{2}}\right]^{n}-2\right\}^{\frac{1}{2}}=\prod_{k=0}^{\frac{n}{2}-1} 2\left(u^{2}+\sin ^{2} \frac{2 k}{n} \pi\right)^{\frac{1}{2}},
\end{gathered}
$$

Equation (1) can be gotten from Theorem 7.

2. When $n$ is odd, it can be seen that the first term of the right hand of Equation (1) is equal to zero, and the second term equals the last term. If $r=0$, to the number of perfect matchings of a graph, the result in Theorem 8 must be the same as Equation (1), so we have following identity: if $m \equiv 0(\bmod 4)$,

$$
\begin{aligned}
& \prod_{t=0}^{m-1}\left\{\left[-\cos \frac{2 t+1}{m} \pi+\left(1+\cos ^{2} \frac{2 t+1}{m} \pi\right)^{\frac{1}{2}}\right]^{n}-\left[\cos \frac{2 t+1}{m} \pi+\left(1+\cos ^{2} \frac{2 t+1}{m} \pi\right)^{\frac{1}{2}}\right]^{n}\right\}^{\frac{1}{2}} \\
& \quad=\prod_{k=1}^{n / 2} \prod_{l=1}^{m} 2\left(\sin ^{2} \frac{2 k-1}{n} \pi+\sin ^{2} \frac{2 l-1}{m} \pi\right)^{\frac{1}{2}} .
\end{aligned}
$$

If $m \equiv 2(\bmod 4)$,

$$
\begin{gathered}
\left\{-\prod_{t=0}^{m-1}\left[\left(-\cos \frac{2 t}{m} \pi+\left(1+\cos ^{2} \frac{2 t}{m} \pi\right)^{\frac{1}{2}}\right)^{n}-\left(\cos \frac{2 t}{m} \pi+\left(1+\cos ^{2} \frac{2 t}{m} \pi\right)^{\frac{1}{2}}\right)^{n}\right]\right\}^{\frac{1}{2}} \\
=\prod_{k=1}^{n / 2} \prod_{l=1}^{m} 2\left(\sin ^{2} \frac{2 k-1}{n} \pi+\sin ^{2} \frac{2 l-1}{m} \pi\right)^{\frac{1}{2}} .
\end{gathered}
$$

3. Turning to the case when $n$ and $r$ are odd, obviously $m$ is even, we find that the determinant of the skew symmetric matrix of the corresponding directed graph is not easy to calculate, hence we pose naturally the problem: how to enumerate perfect matchings of $Q_{m, n, r}$, when $n$ and $r$ are odd. As a continuance, we will consider the lattice on Klein bottle.

4. We still do not known whether graphs $Q_{m, n, r}$ are Pfaffian or not, thought we have enumerated the perfect matchings of it by Pfaffians, it is an interesting problem to be study. 


\section{Acknowledgements}

We are grateful to the referees for providing helpful suggestion.

\section{References}

[1] S. J. Cyvin and I. Gutman, Kekulé structures in Benzennoid Hydrocarbons, Springer Berlin, 1988.

[2] J. Chen and X. chen, Special Matrices, Tsinghua Press, 2001 (in Chinese).

[3] M. E. Fisher, Statistical mecanics of dimers on a plane lattice, Phys. Rev. 124(1961) 1664-1672.

[4] A. Galluccio and M. Loebl, On the theory of Pfaffian orientations. I. Perfect matchings and permanents, Electron. J. Combin. 6, No. 1 (1999).

[5] G. G. Hall, A Graphic Model of a Class of Molecules, Int. J. Math. Edu. Sci. Technol., 4(1973), 233-240.

[6] W. Jockusch, Perfect matchings and perfect squares, J. Combin. Theory Ser. A 67(1994), 100-115.

[7] P. W. Kasteleyn, The statistics of dimers on a lattice I: The number of dimer arrangements on a quadratic lattice, Physica 27(1961), 1209-1225.

[8] P. W. Kasteleyn, Graph Theory and Crystal Physics. In F. Harary, editor, Graph Theory and Theoretical Physics. Academic Press, 1967, 43-110.

[9] D. J. Klein, Resonance in elemental benzenoids, Discrete Appl. Math. 67(1996) $157-173$.

[10] C. H. C. Little, A characterization of convertible $(0,1)$-matrices, J. Combinatorial Theory 18(1975), 187-208.

[11] L. Lovász and M. Plummer, Matching Theory, Ann. of Discrete Math. 29, NorthHolland, New York, 1986.

[12] F. Lin and L. Zhang, Pfaffian orientation and enumeration of perfect matchings for some Cartesian products of graphs, Electron. J. Combin. 16(1), (2009) \#R52.

[13] W. T. Lu and F. Y. Wu, Close-packed dimers on nonorientable surfaces, Physics Letters A 293 (2002) 235-246./

[14] H. N. V. Temperley, M. E. Fisher, Dimer problem in statistical mechanics-An exact result, Philos. Magazine 6 (1961) 1061-1063.

[15] W. McCuaig, N. Robertson, P. D. Seymour, and R. Thomas, Permanents, Pfaffian orientations, and even directed circuits (Extended abstract), Proc. 1997 Symposium on the Theory of Computing (STOC).

[16] H. Narumi and H. Hosoya, Proof of the generalized expressions of the number of perfect matchings of polycube graphs, J. Math. Chem. 3(1989), 383-391. 
[17] J. Propp, Enumeration of Matchings: Problems and Progress, In: New Perspectives in Geometric Combinatorics (eds. L. Billera, A. Björner, C. Greene, R. Simeon, and R. P. Stanley), Cambridge University Press, Cambridge, (1999), 255-291.

[18] N. Robertson, P. D. Seymour, and R. Thomas, Permanents, Pfaffian orientations, and even directed circuits, Annals of Math., 150(1999), 929-975.

[19] G. Tesler, Matchings in graphs on non-orientable surfaces, J. Combin. Theory Ser. B 78(2)(2000), 198-231.

[20] C. Thomassen, Tilings of the torus and the klein bottle and vertex-transitive graphs on a fixed surface. Transactions of the American Mathematical Society. Vol. 323, No. 2(Feb.,1991).

[21] W. Yan and F. Zhang, Enumeration of perfect matchings of a type of cartesian products of graphs, Discrete Appl. Math. 154(2006), 145-157.

[22] W. Yan, Yeong-Nan Yeh, F. Zhang, Dimer problem on the cylinder and torus, Physica A., 387(2008), 6069-6078.

[23] F. Zhang and W. Yan, Enumeration of perfect matchings in a type of graphs with reflective symmetry, MATCH Commun. Math. Comput. Chem., 48(2003), 117124. 